\title{
La ofensiva de Ignaz Semmelweis contra los miasmas ineluctables y el nihilismo terapéutico
}

\author{
ta ofensiva de lgnaz Semmelweis contralos \\ miasmas ineluetables y el nihillismo terapéutieo
}

\author{
Octavio Martínez • CIUdAd XXXX
}

\begin{abstract}
Resumen
A Semmelweis, la posteridad sólo le reconoce la introducción del lavado antiséptico de manos para reducir la infección hospitalaria, lo acusa de haber sido incapaz de superar las dificultades epistémicas para levantar el statu quo imperante, se lo denuesta por defender con fervor sus ideas, por su tosquedad y torpeza en las relaciones con sus colegas, y por la rudeza de sus escritos. Su cometido se centraba en desarrollar prácticas obstétricas seguras, valorativas de la vida de la madre y cumplidoras del imperativo ético para el médico, por sobre todo no hacer daño. La deuda que se tiene con Semmelweis es el reconocimiento de su altura moral. (Acta Med Colomb 2014; 39: 00-00).

Palabras clave: Semmelweis, miasmas, nihilismo terapéutico, "primum non nocere"
\end{abstract}

\begin{abstract}
Posterity only reeognized to Semmelweis the introduction of antiseptie hands washing to reduce nosoemial infeetions. He is aeetused of had not been able to overeome epistemie diffieulties to replaee aetual statts quo. He has been disdained as a result of the defense of his ideas with ardeney, and beeause coarseness and clumsiness relationships with colleagues, and the rudeness of his writings. His aim was foeused on developing safe obstetrie praetiees, with respeet for mother life and eompliant the ethieal imperative, above all do no harm, by doetors. Debt with Semmetweis is the reeognition of his moral height. (Aeta Med Colomb 2014; 39:-00-00).
\end{abstract}

Key words: Semmetweis, miasmas, therapettic nihitism, "primam non nocere"
Dr. Octavio Martínez Betancur: Profesor Asociado, Departamento de Medicina Interna, Facultad de Medicina, Universidad Nacional de Colombia. eiudad $x x x$ Correspondencia. Dr. Octavio Martínez Betancur eiudadxxx

E-mail: omartinezb@unal.edu.co

Recibido: 1/IV/2013 Aceptado: 16/X/2013

\section{Introducción}

Ignaz Semmelweis, proclamado fuera de su tiempo el precursor del lavado antiséptico de manos para disminuir infecciones nosocomiales, no alcanzó a rebatir la teoría miasmática de las epidemias imperante en su época ni a completar una teoría comprensiva sobre la causa común necesaria para la fiebre puerperal. Sus observaciones clínicas, sus juiciosos estudios estadísticos y sus inferencias causales no fueron revolucionarios en el sentido de ruptura paradigmática, sino una revuelta contra el "nihilismo terapéutico" que permeaba todos los estamentos del Imperio de los Habsburgo, tanto en lo político y cultural como en lo médico y científico. Su cometido se centraba en desarrollar prácticas obstétricas seguras, valorativas de la vida de la madre y cumplidoras del imperativo ético para el médico, por sobre todo no hacer daño.

Los datos tabulados y analizados por Semmelweis (1) fueron desechadas por sus contradictores, quienes respon- dían a una larga tradición de dogmas no cuestionados, rehusando aceptar la evidencia experimental que no se ajustaba con sus prejuicios. El propósito de este estudio es relacionar el logro cognitivo de Semmelweis con las circunstancias culturales, políticas, económicas y académicas dentro de las cuales gestó su delirante y apasionado trabajo, partiendo del supuesto que la actividad científica está permeada por los valores de la sociedad en que interactúa el investigador. El reconocimiento de la altura moral de Semmelweis es la deuda que la comunidad académica debe saldar con su obra, que fue su vida.

\section{Pervivencia de los miasmas}

Durante todo el siglo XVIII, las cuatro principales explicaciones para las epidemias fueron, (i) la "constitución epidémica" de la atmósfera, (ii) las condiciones "miasmáticas" locales ocasionadas por el clima, las estaciones, los enfermos y la materia orgánica putrefacta, (iii) el contagio, y 
(iv) la variación en la resistencia vital de los sujetos, presunción vaga para explicar casos individuales extraordinarios. Para consideraciones prácticas, los miasmas y el contagio eran las dos teorías en la cuales se basaban las medidas preventivas (2).

Al comienzo del siglo XVIII, la transmisión aérea ya fuese a cortas o largas distancias, fue considerada por la profesión médica de primera importancia, y de segunda, el contagio entre personas, fuese directo o indirecto a través de objetos inanimados. La infección se pensaba debida a la transferencia de miasmas que contenían partículas envenenadas de la exhalación pútrida de la persona enferma a la persona saludable. La resistencia de los médicos a la teoría del contagio se fortalecía al observar la diseminación de la plaga de país a país y de puerto en puerto, y la experiencia decía que se daban muchas epidemias que el contagio entre personas no explicaba, como casos de epidemias en nuevos localidades que no habían tenido contacto con ninguna persona enferma y que desaparecían tan inexplicablemente como se presentaban, sin diseminación ulterior. Así, el énfasis durante todo el siglo XVIII fue la ventilación de los ambientes hospitalarios, cárceles, barcos, instalaciones militares y demás espacios cerrados, en prevención de epidemias de infección a través de la transmisión aérea (miasmas) $(2,3)$.

El nombre de fiebre puerperal apareció en 1720 y las primeras descripciones se dieron alrededor de 1740. Hasta la primera mitad del siglo XVIII los partos eran atendidos por comadronas. El nacimiento no formaba parte del cuidado médico y con muy pocas excepciones, las parteras no llevaban registros. Fue la participación del médico en el parto y especialmente la fundación de hospitales obstétricos en la segunda mitad del siglo XVIII lo que forzó la atención en la enfermedad. La fiebre puerperal ocurría en dos formas, esporádica y epidémica en ciudades y hospitales. La tasa de mortalidad en casos esporádicos era cercana a 30\%, tan altas como $80 \%$ al inicio de las epidemias y amainaba en la medida que la epidemia cedía (4-7). La prevalencia de la enfermedad junto con la inefectividad de las medidas preventivas, sugerían que la enfermedad era epidémica, y las enfermedades epidémicas estaban más allá del control humano. La teoría miasmática de la fiebre puerperal tenía apoyo casi universal, inculpando a las impropias condiciones atmosféricas la principal causa de la rápida diseminación de la infección una vez instalada en las maternidades hospitalarias, contra la cual no podía haber defensa $(2,8,9)$.

El objetivo del diseño del Hospital General de Viena, "casa de almas" para pobres y soldados con capacidad para 2000 personas, fundado en 1784 e instalado en un edificio ya construido con 25 años de antigüedad, era la adecuada circulación de aire de conformidad con la entonces vigente teoría miasmática de la infección. La Clínica de Maternidad del Hospital General de Viena, dirigida por Johann Klein, profesor jefe de obstetricia de gran importancia para la negativa y hostil recepción de la ideas de Semmelweis, ocupaba el espacio entre el hospital de fiebres y el departamento quirúrgico. Por estándares de construcción de la época, constaba de ocho salas amplias intercomunicadas, con 20 camas cada una. Cada sala tenía diez ventanas altas a ambos lados para permitir la ventilación cruzada por sobre las cabezas de los pacientes, y se instalaron numerosos ventiladores, aún sobre las camas de los pacientes para eliminar los efectos del "aire corrupto" y diluir los efluvios putrefactos. El mecanismo de disposición de excretas se diseñó de tal manera que previniera el escape de olores. El énfasis se ponía en la ventilación y en la distancia que separaba los pacientes $(10,11)$.

Semmelweis, quien en 1846 se posesiona como Asistente Provisional y asciende al año siguiente a Asistente en la Clínica del profesor Klein, centra sus observaciones en encontrar y superar las causas de la excesiva mortalidad en la Primera Clínica, con tres consideraciones definitivas para su investigación $(12,13)$ :

1. En 1820, la aproximación anatómica al estudio de la medicina era el estándar en Viena. Para un mejor entendimiento de la enfermedad era necesario realizar disecciones de cadáveres constantemente. Los estudiantes de medicina extranjeros venidos a adquirir experiencia en la clínica de obstetricia más grande de Europa, asignados en número de 20 a 30 para asistir el trabajo de la Primera Clínica, caminaban desde la sala de autopsias directamente a las áreas de trabajo y atención del parto. Semmelweis notó que antes de 1820 , las tasas de mortalidad en el Hospital General de Viena eran mucho menores que $1 \%$ anual.

2. En 1833, el Hospital General de Viena dividió su servicio obstétrico en dos unidades, cada una compuesta tanto por médicos como por parteras. Durante ese tiempo, las tasas de fiebre puerperal eran bastante parecidas, cercanas a $6 \%$ anual y en algunos meses eran tan altas como $25 \%$ a 30\%. En 1840 el personal de las unidades fue reasignado. La Primera Clínica la componían exclusivamente médicos y la Segunda Clínica únicamente parteras. Este cambio generó tasas discordantes de fiebre puerperal con aumento significativamente mayor en la Primera Clínica. Este hecho condujo a que Semmelweis desestimara la teoría epidémica de las influencias atmosféricas contra las cuales no había defensa. Las dos Clínicas estaban sujetas a las mismas influencias atmosféricas; si la enfermedad fuera epidémica, habría ocurrido con la misma frecuencia en ambas Clínicas y, además, las tasas de sepsis puerperal en mujeres que tenían sus partos en las calles no serían tan bajas, mucho menores que las que parían en el Hospital. Estos hechos convencieron firmemente a Semmelweis que la enfermedad debía ser endémica (9).

3. No fue el estudio sistemático de las tasas de mortalidad, la observación de los pacientes ni el trabajo diligente en la sala de disección lo que permitió a Semmelweis el reconocimiento de la causa de la fiebre puerperal, sino la muerte en marzo de 1840 de su amigo Jakob 
Kolletschka, profesor de medicina forense. Kolletschka murió de "piemia del patólogo" entidad reconocida que se desarrollaba a partir de una herida menor o no reconocida en las manos durante una disección, hasta que una veta eritematosa, dolorosa a lo largo del brazo anunciaba la presencia de una infección potencialmente fatal. Cuando Semmelweis estudió los resultados de la autopsia realizada al cuerpo de Kolletschka, reconoció que los hallazgos eran idénticos a los característicamente encontrados en madres muertas de fiebre puerperal. La infección masiva de Kolletschka había sido causada por la introducción de "materia cadavérica" en una pequeña herida con bisturí. Semmelweis concluyó que la materia cadavérica también debía ser la causa de la fiebre puerperal. Justo como el bisturí de disección introduce materia cadavérica al interior del torrente sanguíneo del patólogo, las manos contaminadas de los médicos examinadores acarrean materia cadavérica de la sala de autopsia a la mujer en parto (14-21).

Semmelweis también había observado que cuando las mujeres morían de fiebre puerperal, sus recién nacidos, tanto hombres como mujeres, algunas veces morían de una fiebre con las mismas consecuencias anatomopatológicas de la fiebre puerperal. El paso decisivo en el razonamiento de Semmelweis fue la conclusión que el estado puerperal no era una condición necesaria para la presentación de la enfermedad. Así las cosas, su presunción fue que varios casos de una enfermedad particular debían mostrar una causa común. En un ensayo publicado en 1858 dijo explícitamente, "Para que pueda ocurrir fiebre puerperal, es condición sine qua non que sea introducida materia cadavérica en los genitales", indicación directa que en concepto de causalidad en Semmelweis está vinculado con el de necesidad $(9,22)$.

"Kolletschka también tenía la enfermedad, entonces la fiebre puerperal no es una especie de enfermedad; en vez de ello, es un tipo de piemia". No había temor en Semmelweis en la recategorización que pudiera derivarse de su propuesta. El concepto de fiebre puerperal podía desaparecer (9). El conflicto ontológico entre Semmelweis y sus colegas estaba parcialmente sustentado en desacuerdos sobre la manera en la cual debían definirse y clasificarse las enfermedades. Los hallazgos morfológicos eran decisivos. No se excluía la posibilidad que cada enfermedad tuviera varias causas no relacionadas, y Semmelweis no buscaba explicar todas las causas de fiebre puerperal, sino únicamente encontrar y superar las causas de la excesiva mortalidad en la Primera Clínica. La aproximación de Semmelweis a la intervención de lavado de manos, así como en el resto de la situación experimental, pertenecen al realismo de las consecuencias empíricas y no al reflejo de la hipótesis causal por sí misma (22).

En oposición con las autoridades médicas, Semmelweis afirmaba que únicamente su doctrina era consistente con el cambio en las estadísticas de mortalidad en el Hospital General de Viena. Su teoría fue rechazada por los líderes de opinión más influyentes, a pesar de la evidencia experimental clara, considerada como una fluctuación estadística. Después de todo, existía considerable variación en las tasas de mortalidad. Su teoría fue acusada de únicamente producir resultados palpables, sin ningún soporte racional derivado de la doctrina anatómica prevalente, la cual postulaba que toda enfermedad debe resultar de cambios orgánicos en la estructura tisular. El concepto de materia orgánica descompuesta era ininteligible en tal doctrina, y las medidas antisépticas no se ajustaban con ninguna teoría disponible. Más aún, el lavado de manos parecía ser demasiado simplista para responder a epidemias supuestamente gestadas por fuerzas cósmicas ineluctables que ostensiblemente absolvían de responsabilidades a los médicos por el destino de sus pacientes. Semmelweis no se preocupó por buscar pruebas teóricas de su teoría. Él había llegado a su descubrimiento por medios empíricos y concentró toda su energía en la aplicación práctica para salvar mujeres de la amenaza de la muerte $(17,23)$.

En el mundo de Kuhn, durante un periodo de ciencia normal, un investigador no puede adelantar conjeturas arbitrarias sino únicamente conjeturas que sean compatibles con el paradigma dominante. Si un científico adelanta una conjetura que contradice el paradigma dominante, es probable que sea considerada como un absurdo. En algunos casos, tal hipótesis puede marcar el comienzo de una revolución, pero, aun si la hipótesis es vindicada con el tiempo, es probable que en principio sea fuertemente atacada por la comunidad científica. Lo anterior sugiere una razón kuhninana para la falla de Semmelweis. Su práctica tenía necesidad de ser justificada por una teoría, y la teoría que él adelantó fue rechazada porque contradecía el entonces paradigma dominante concerniente con la causa de la enfermedad (24).

La mayoría de los contemporáneos de Semmelweis, consideraban la fiebre puerperal como una enfermedad de carácter epidémico relacionada con la calidad del aire, mientras otros la entendían como contagiosa, y que afectaba fundamentalmente, si no exclusivamente, a embarazadas y parturientas a través de la alteración mórbida del útero (21). Existían pues dos teorías principales para explicar las causas de fiebre puerperal: la teoría miasmática y la teoría del contagio. La existencia de dos teorías diferentes sugiere un periodo pre-paradigmático, pero estas teorías no eran sustentadas por escuelas contendoras de pensamiento en medicina. Al contrario, casi todos los médicos consideraban que ambas teorías eran correctas y que las enfermedades eran explicadas en algunos casos por una de las teorías, en otros casos por la otra teoría, y aún en otros casos por alguna combinación de las dos teorías. Lo que se ve entonces no es un periodo pre-paradigmático, sino lo que puede describirse como un paradigma compuesto (24).

Tras haberse lavado cuidadosamente las manos según sus propias indicaciones de desinfección y examinado primero a una parturienta que sufría cáncer uterino ulcerado, examinó a otras 12 mujeres de la misma sala después de un 
lavado rutinario sin desinfección. Al morir once de ellas de fiebre puerperal, la conclusión de Semmelweis fue que no sólo la materia cadavérica era el agente de la fiebre sino también "materia orgánica pútrida procedente de organismos vivos" (21). Para Semmelweis la fiebre puerperal no era una enfermedad contagiosa. El contagio a partir de un sujeto con una enfermedad contagiosa acarrea únicamente la misma enfermedad a otros sujetos. No era el caso de la fiebre puerperal, porque podía ser causada por medio de otras enfermedades como carcinoma del útero y exhalación de una rodilla con osteomielitis (24). Semmelweis postula entonces una única causa necesaria y común (universal) para la fiebre puerperal, la "materia orgánica pútrida", haciendo de ella la base para la caracterización de la enfermedad, en oposición a la clasificación tradicional basada en síntomas clínicos y hallazgos patológicos. El paradigma reduccionista anatómico-patológico en el que se fundamentaba la nosología de la época no mostraba anomalías que vislumbraran su resquebrajamiento, y por supuesto, pocos vieron que Semmelweis había identificado una causa necesaria para la fiebre puerperal. Lo expresa Céline, “...las energías del investigador más audaz, del más preciso, se detenían en la Anatomía Patológica" (25). Más aún, Semmelweis carecía de una teoría explicativa que diera cuenta de la naturaleza de la "materia cadavérica" o "materia pútrida", y de la relación causal de ésta con la fiebre puerperal (21). El vacío teórico de Semmelweis sobre la naturaleza de la "materia" causante de la enfermedad se atribuye a su marcada preocupación porque su método de prevención fuera utilizado para salvar vidas. Semmelweis enfatizaba la importancia de los datos numéricos en sus investigaciones: "La enfermedad puerperal sigue siendo un misterio, únicamente el número de muertos es un hecho palpable" (23). Su teoría, aunque fundamentada en la evidencia estadística, no podía diferenciar entre agente transmisor y agente patógeno. La hipótesis de Semmelweis identificaba en la "materia pútrida" al agente transmisor, y justificaba el lavado de manos con agentes clorados para romper la cadena causal, pero no informaba sobre la naturaleza material de tal entidad, ni explicaba su acción patógena en el organismo (21). En su momento, esta "materia" ínfima que creía provocar la infección puerperal era imponderable, sólo reconocible por el olor. "Desodorizar las manos", decidió Semmelweis (25).

El prolongado debate médico sobre la naturaleza de la fiebre puerperal se resolvió en contra de la teoría del contagio, puesto que se rehusaba creer que los médicos con sus aparentes manos limpias pudieran ser agentes de la muerte $(17,26)$. Semmelweis fue incapaz de convencer a sus colegas para mantener su teoría. Aunque mantenía copiosas notas, se rehusó a informar sus hallazgos en revistas médicas, en parte porque era consciente de sus pocas destrezas lingüísticas y sentía temor de no poder articular suficientemente bien sus pensamientos. La mayoría de las publicaciones iniciales sobre el tema fueron hechas por sus colegas (19). El trabajo de Semmelweis fue primeramente anunciado en 1847 por
Ferninand Hebra y Joseph Skoda, anuncio que no contenía sugerencia alguna de que cada caso de fiebre puerperal era debido a una causa común. Aquellos quienes entendieron las posturas de Semmelweis, las rechazaron; otros las ignoraron aunque aceptaron el lavado de manos con cloro. En 1849 Skoda presentó los resultados de Semmelweis ante el Comité de Estudios de la Escuela Médica de Viena con la sugerencia que la Facultad de Medicina realizara una investigación. Esta propuesta fue considerada como una afrenta abominable por parte del profesor Klein, quien se opuso a la investigación, y el Ministro de Educación denegó la autorización para esfuerzos de investigación dirigidos por la Facultad. En 1849 no se le renovó el contrato de Asistente a Semmelweis (12). Semmelweis no era considerado por los directivos de la Facultad de Medicina de la Universidad de Viena como un académico riguroso del talante de Rokitansky o Skoda, sino más bien un clínico con práctica en obstetricia. La manifiesta hostilidad de Klein hacia Semmelweis se enmarca dentro de los celos profesionales de Klein por el éxito terapéutico de Semmelweis, resentimiento de Klein por creer que las estadísticas recogidas por Semmelweis perjudicaban la imagen de la clínica universitaria e incluso simple antipatía personal y diferencias ideológicas. Por otra parte, Klein enseñaba todavía en Viena la teoría láctea como causa de fiebre puerperal (21).

Entre 1850 y 1861 no existe evidencia documental que alguien en Europa aceptara el postulado de Semmelweis de haber encontrado una causa necesaria universal para la enfermedad. Hacia 1860 los médicos que conocían la teoría por vaguedades de segunda mano, asumieron que había sido desacreditada desde 1840. Cuando finalmente en 1861 publica The Etiology, Concept, and Prophilaxis of Childbed Fever, sus ideas ya se habían distorsionado, se tenía una visión errónea acerca de su trabajo, y pocos se molestaron en leer la exposición completa de la teoría (9).

\section{Contra el nihilismo terapéutico}

Durante la administración de Klemens, príncipe de Metternich, bajo el reinado del emperador Francisco I (1792-1835) y de su hijo Fernando I de Austria (18351848), se fomentó la resignación política y la represión del racionalismo. Se permitía a los altos funcionarios del Estado centralizado ejercer toda clase de arbitrariedades empleando la censura, la policía secreta y el reglamentismo como elementos disuasorios de la actividad política y la innovación científica. Nobleza y clases medias bajas conspiraban para oponerse a las innovaciones y animaban a la corte a mantener el statu quo. Tras la revolución de marzo de 1848, con la renovación administrativa impuesta por el emperador Francisco José (1848-1916), se superó paulatinamente la inercia burocrática, y el imperio de los Habsburgo empieza a promover a investigadores de talento y fortalecer la investigación universitaria (27)

El privilegio más dañino de la aristocracia era su capacidad para hacer y deshacer carreras profesionales. Como 
los miembros de la sociedad cortesana otorgaban favores a los dirigentes del aparato del Estado, cualquier proyecto requería un respaldo preliminar de la corte si quería conseguir la aprobación administrativa. Mediante la institución de la Protektion (proteccionismo), archiduques, príncipes y un puñado de profesores encumbrados ejercían su influencia sobre prácticamente todas las facetas de la vida pública. La aristocracia se aprovechaba de su autoridad sin traza alguna de organización ni liderazgo, impidiendo la innovación, negando apoyo a todo de tipo de iniciativa novedosa. La Protektion ejerció su influencia no sólo en las cátedras universitarias, sino, de hecho, en la totalidad de las instituciones culturales. Los profesores universitarios eran funcionarios, servidores personales del emperador, que podía ejercer el veto en cualquier tipo de nombramiento. Gracias al riguroso control que el Ministerio de Educación imponía así sobre los nombramientos del profesorado, el sistema de Protektion campaba por sus respetos (27).

Johann Klein, profesor jefe de obstétrica de la Primera Clínica, superior de Semmelweis, era una figura de moda tanto en el mundo médico como en los círculos aristocráticos. Era considerado más vistoso que brillante, buen católico, hombre de familia y un efusivo patriota, combinación austríaca de resignación política y capacidad de intriga burocrática (10). El nombramiento inicial de Semmelweis como maestro de cirugía en noviembre de 1846 se vio truncado al no disponerse de ninguna vacante en las cátedras disponibles, lo que lo impacientó y obligó a aceptar la cátedra de obstetricia bajo la dirección de Klein, cátedra otorgada una vez cumplidos los requisitos académicos por el Ministro de Educación anta la mediación de sus maestros Rokitansky y Skoda. La protección que Skoda, médico de cabecera de la familia imperial, le brindaba a Semmelweis en el mundo cortesano de su tiempo, no fue suficiente para que toda la desastrosa influencia y rabiosa obstrucción de Klein en contra de las ideas y procedimientos propuestos por Semmelweis, terminaran en una animadversión general por parte de sus colegas, su destitución y perentoria expulsión de Viena, en marzo de 1849 (25).

Dado el gusto vienés por la desesperanza cultural, la tendencia general de la intelectualidad médica de la época era anteponer la formulación del diagnóstico a la administración de la terapia. Esa especie de abstinencia terapéutica es conocida con el nombre decimonónico de nihilismo terapéutico, procedente de la Facultad de Medicina de Viena. Como lo demuestran los pioneros del diagnóstico empírico como Rokitansky o Skoda, hacia 1850 y durante diez o veinte años más se creía necesario centrar la investigación sanitaria en los síntomas de las enfermedades y no en las correspondientes terapias. Durante toda una generación, estos puristas de la experimentación científica hicieron pasar a un segundo plano la investigación sobre los procedimientos curativos para, a cambio, dedicarse a examinar las historias clínicas en condiciones impolutas, sin incurrir en vanos intentos de curación. Los gigantes de la Escuela de Medicina vienesa, preferían el diagnóstico post mórtem a la terapia a su debido tiempo, sacrificando al paciente en aras de la ciencia (27). En esta matriz disciplinar se inscribe el trabajo de Semmelweis, visto por los adeptos al nihilismo terapéutico como algo impropio de un profesional médico, por lo que su descubrimiento de atenuar la frecuencia de fiebre puerperal con el lavado de manos, no consiguió imponerse como una verdad simple sobre el mundo médico de su tiempo. Además, si las mujeres mueren de fiebre puerperal, es el destino, la fatalidad, la expiación, la venganza de Dios por su desvergüenza. Salvarlas con un lavado de manos antes del examen obstétrico, todo un despropósito (28).

La creación de la cultura conocida como Biedermeier (1815-1848) que animaba a las clases medias austríacas a emular los intereses estéticos que anteriormente habían atraído a la aristocracia, favoreció lo que se conoce como el "hombrecito". El héroe popular celebrado era el ciudadano sufridor, normalmente de mediana edad y clase media baja, que acepta su moderada suerte y cree en la Providencia. Planteado como la personificación del sentimiento de resignación que sentían los ciudadanos hacia la burocracia administrativa y la aristocracia imperial, el "hombrecito" demostraba cómo los humildes podían gozar de la Creación obedeciendo las leyes divinas. El término "hombrecito" designa a los intelectuales de origen modesto que manifestaban una humildad fuera de lo común a fin de disimular sus aspiraciones creativas. La administración Biedermeier mostraría un nulo interés por los inventores del período. Resistencia burocrática hacia la innovación. La renuncia a lo mundano desembocó a veces en la fascinación hacia la muerte. El rechazo de los intelectuales austriacos para proponer remedios-nihilismo terapéutico-reforzó la resignación característica del Biedermeier (27). Semmelweis siempre se vio asimismo como un marginado que, sin hablar el lenguaje correcto, falto de diplomacia, inseguro y carente de la habilidad necesaria para escribir científicamente, publicar y defender su trabajo, golpeaba las puertas del panteón académico de la Escuela de Medicina de Viena $(12,19)$. Semmelweis desarrolló un apremiante sentido del tiempo y quiso establecer su teoría en 1848 como una ola de revolución liberal que barriera toda Europa así como el reconocimiento oficial del establecimiento médico, propósito difícil de lograr siendo húngaro de talante izquierdista, involucrado en disturbios revolucionarios, en un mundo académico autoritario, conservador, germano hablante donde sus opiniones como asistente no se preferían a las de un profesor austríaco $(17,21,24)$.

\section{"Primum non nocere"}

En la perspectiva médica reduccionista de los anatomopatólogos vieneses, el flujo dialógico de la relación médico paciente era superado por el imperativo científico de obtener información biológica acerca de la enfermedad. El centro de atención del clínico no era el bienestar de las pacientes sino entender los cambios biológicos involucrados en la 
enfermedad. Se violentaba la humanidad de las madres en aras de un utilitarismo de acto en favor de la ciencia y la humanidad sin importar la autonomía de ellas. Las decisiones médicas no se adherían a juicios reflexivos morales en relación con el deber de elegir la decisión concertada que tuviera las consecuencias más positivas las pacientes. Madres solteras olvidadas por sus familias, degradadas socialmente y abandonas en las calles donde sobrevivían de limosnas y en muchos casos de la prostitución, absolutamente miserables, iletradas y con gran temor de Dios y reverencia por los médicos, sin otra alternativa que la asistencia dada por la sanidad pública, debían resignarse al paternalismo que el estamento médico consideraba como necesario y moralmente aceptable. La excelencia moral de los médicos estaba por encima de toda duda. En la discusión del acto médico no contaban la culpa legal y menos la mortificación moral ante las muertes de pacientes. Antes por el contrario, se inculpaba a las propias madres por dichos fracasos. La autoestima del médico se mantenía incólume no obstante la humillación de las pacientes (29-32).

Contiguo al pabellón de maternidad de Klein en el Hospital General de Viena, de idéntica construcción, se alzaba el pabellón atendido por parteras bajo la dirección de profesor Bartch. La admisión de las mujeres se realizaba por turnos de 24 horas en uno u otro pabellón, a partir de las cuatro de la tarde. Ya era conocido en Viena que el riesgo de muerte tras ingresar al pabellón de Klein equivalía a la certeza. Semmelweis fue testigo de las súplicas con quejidos y llanto de mujeres que imploraban ser admitidas en el pabellón de Bartch en nombre de su vida y la de sus otros hijos, sin otra respuesta que ser arrastradas por la fuerza hacia una muerte segura $(20,25)$. Pareciera que en toda Viena el único que se mortifica con el tributo mortal a pagar por la maternidad es Semmelweis, y lo confiesa en carta a su amigo Markusovsky: “...debo confesarle que mi vida ha sido infernal, que la idea de la muerte de mis pacientes me ha resultado siempre insoportable, sobre todo cuando se cuela entre las dos grandes alegrías de la existencia, la de ser joven y la de dar la vida" (25). Como compañía de su melancólica soledad le llega la noticia que en Kiel, un profesor llamado Michaelis, se ha suicidado tras la desesperación que le significó sentirse responsable de la muerte por fiebre puerperal de una de sus primas por él atendida, sin el lavado antiséptico de manos propuesto por Semmelweis, habiendo atendido previamente a otras mujeres muertas de infección puerperal. Semmelweis se resiente con el silencio de sus colegas. Ni Virchow en Alemania ni Dubois en París, ambos conminados a acompañar a Semmelweis en su batalla por combatir la muerte por fiebre puerperal, hacen eco de los resultados logrados con el lavado antiséptico de manos (25).

Semmelweis acepta finalmente su frustración y arroja una avalancha de ácidos panfletos y cartas abiertas donde acusaba a sus críticos de haber masacrado madres e hijos. Cita el nombre de quienes consideraba sus enemigos, y los denuncia "ante Dios y el mundo" como Nerones médicos culpables de homicidio intencionado (17). “iAsesinos! Así es como llamo yo a todos los que se oponen a las reglas que he prescrito para evitar la fiebre puerperal. Frente a ellos me levanto como adversario resuelto, ¡como debe levantarse un hombre ante los cómplices de un crimen! Para mí, no hay otro modo de tratarles que como asesinos... No son las maternidades lo que hay que cerrar para que terminen los desastres que ocurren en ellas, son los obstetras los que deben salir de ellas, pues son ellos los que se comportan como auténticas epidemias..." (25).

A Semmelweis, la posteridad sólo le reconoce la introducción del lavado antiséptico de manos para reducir la infección hospitalaria, lo acusa de haber sido incapaz de superar las dificultades epistémicas para levantar el statu quo imperante, se lo denuesta por defender con fervor sus ideas, por la tosquedad y torpeza en las relaciones con sus colegas, y por la rudeza de sus escritos. La deuda que se tiene con Semmelweis es el reconocimiento de su altura moral.

\section{Referencias}

1. Semmelweis I. The etiology, concept, and prophylaxis of childbed fever. In Buck C, Llopis A, Najera E, Terris M. The challenge of epidemiology. Issues and selected readings. Scientific Publication $\mathrm{N}^{\circ}$ 505. Washington: OPS/OMS; 1998: 46-59.

2. Ayliffe GAJ, English MP. Hospital infection: From miasmas to MRSA. Cambridge: Cambridge University Press; 2003. 274 p.

3. Wood WB. From miasmas to molecules. New York: Columbia University Press; 1961. $100 \mathrm{p}$

4. Weissmann G. Puerperal priority. Lancet 1997; 349: 122-125.

5. London I. The tragedy of puerperal fever. Health Libraries Reviews 1998; 15: 151-156.

6. Adriaanse AH, Pel M, Bleker OP. Semmelweis: the combat against puerperal fever. Eur J Obst Gynecol \& Repr Biol 2000; 90: 153-158.

7. Gould IM. Alexander Gordon, puerperal sepsis, and modern theories of infection control-Semmelweis in perspective. Lancet 2010; 10: 275-278.

8. Carter KC. Semmelweis and his predecessors. Medical History 1981; 25: 57-72.

9. Carter KC. Ignaz Semmelweis, Carl Mayrhofer, and the rise of germ theory. Medical History 1985; 29: 33-53.

10. Dormandy T. Four creators of modern medicine. Moments of truth. West Sussex: Wiley; 2003. 563 p.

11. Fleming JB. Puerperal fever: The historical development of its treatment. Proc $R$ Soc Med 1966; 59: 341-345.

12. Miller PJ. Semmelweis. Infect Control 1982; 3: 405-409.

13. Sepkowitz KA. Cassandra and Ignaz Semmelweis. Intern J Infect Dis 1996; 1: $57-58$.

14. Elek SD. Semmelweis and the Oath of Hippocrates. Proc R Soc Med 1966; 59: 346-352.

15. Henao G. La fiebre puerperal. La lucha de I. F. Semmelweis en la génesis de un nuevo paradigma. Iatreia 1999; 12: 149-156.

16. Miranda M, Navarrete L. Semmelweis y su aporte científico a la medicina: Un lavado de manos salva vidas. Rev Chil Infect 2008; 25: 54-57.

17. Magner LN. A history of medicine. $2^{\text {nd }}$ ed. Boca Raton: Taylor \& Francis Group; 2005. $624 \mathrm{p}$.

18. Noakes TD, Borresen J, Hew-Butler T, Lambert MI, Jordaan E. Semmelweis and the aetiology of puerperal sepsis 160 years on: and historical review. Epidemiol Infect 2008; 136: 1-9.

19. Ligon BL. Historical moments in the recognition of hand hygiene for control of infections: a short biography of Ignaz Philipp Semmelweis. Sem Ped Infect Dis 2001; 12: 154-159.

20. Nuland SB. El enigma del doctor Ignác Semmelweis. Fiebres de parto y gérmenes mortales. Barcelona: Antonio Bosch; 2003. 154 p.

21. López JA. El triunfo de la antisepsia. Un ensayo en filosofía naturalista de la ciencia. México, D.F: Fondo de Cultura Económica; 2008. 212 p.

22. Persson J. Semmelweis's methodology from the modern stand-point: intervention studies and causal ontology. Studies Hist \& Philos Biol Biom Scie 2009; 
40: 204-209.

23. Buyse M. A biostatistical tribute to Ignaz Philip Semmelweis. Stat Med 1997; 16: $2767-2772$.

24. Gillies D. Hempelian and Kuhninan approaches in the philosophy of medicine: the Semmelweis case. Studies Hist \& Philos Biol Biom Scie. 2005; 36: 159-181.

25. Céline LF. Semmelweis. Barcelona: Marbot Ediciones; 2009. 118 p.

26. Charles D, Larsen B. Streptococcal puerperal sepsis and obstetric infections: A historical perspective. Rev Infect Dis 1986; 8: 411-422.

27. Johnston WM. El genio austrohúngaro. Historia social e intelectual (1848-1938). Oviedo: KRK Ediciones; 2009. 1147 p.

28. Soller P. Nacimiento de Céline. En: Céline LF. Semmelweis. Barcelona: Marbot Ediciones; 2009: 119-126.
29. Restrepo LC. Violencia médica. [revisado el 23 de julio de 2011]. Disponible en: http://www40.brinkster.com/celtiberia/violenciamedica.html.

30. Wulff HR, Pedersen SA, Rosenberg R. Introducción a la filosofía de la medicina. Madrid: Triacastela; 2002. 307 p.

31.Pauustinen R. The clinical encounter as the core of medicine. In: Evans M Louhiala P, Puustinen R. Philosophy for Medicine. Applications in a clinical context. Oxford: Radcliffe Medical Press; 2004: 17-28.

32. Stempsey W. Guilt, shame and medicine. In: Evans M, Louhiala P, Puustinen R. Philosophy for Medicine. Applications in a clinical context. Oxford: Radcliffe Medical Press; 2004: 47-63. 